\title{
X-ray-induced Luminescence Properties of Nd-doped $\mathrm{GdVO}_{4}$
}

\author{
Masaki Akatsuka, Hiromi Kimura, Daichi Onoda, Daiki Shiratori, \\ Daisuke Nakauchi, Takumi Kato, Noriaki Kawaguchi, and Takayuki Yanagida
}

Division of Materials Science, Nara Institute of Science and Technology, 8916-5, Ikoma, Nara 630-0192, Japan

(Received January 31, 2021; accepted May 18, 2021)

Keywords: scintillation, single crystal, near-infrared, dosimetric application

Nd-doped $\mathrm{GdVO}_{4}$ single crystals were synthesized and their photoluminescence and scintillation properties were evaluated. The Nd-doped samples showed scintillation due to the $4 \mathrm{f}-4 \mathrm{f}$ transitions of $\mathrm{Nd}^{3+}$ with peaks at around 900,1060 , and $1320 \mathrm{~nm}$. In addition, we evaluated the relationship between the scintillation signal intensity and the X-ray exposure dose rate for dosimetric application. All the Nd-doped samples demonstrated high sensitivity with a dynamic range from 0.006 to $60 \mathrm{~Gy} / \mathrm{h}$.

\section{Introduction}

Scintillators are functional materials that convert absorbed radiation energy into thousands of low-energy photons and emit UV, visible, and near-infrared (NIR) light immediately. ${ }^{(1)}$ The range of applications of scintillators is very wide, for example, security, ${ }^{(2)}$ medical imaging, ${ }^{(3)}$ cosmic ray detection, ${ }^{(4)}$ and environmental monitoring. ${ }^{(5)}$ Various performances are required for scintillating materials such as high light yield, fast decay, high effective atomic number, low afterglow level, and chemical stability. However, there are no perfect materials satisfying all these requirements. Thus, suitable scintillators are selected according to their purpose, and many scintillator materials have been developed with different chemical compositions such as crystals,,${ }^{(6,7)}$ ceramics, ${ }^{(8)}$ glasses,${ }^{(9,10)}$ plastics, ${ }^{(11,12)}$ and organic-inorganic compounds. ${ }^{(13)}$

Because scintillators are usually used in combination with photomultiplier tubes (PMTs), which have sensitivity in the UV-visible range, scintillators emitting UV-visible light have been developed. On the other hand, in recent years, scintillators emitting NIR photons have attracted much attention since NIR photons have unique characteristics. NIR photons (700-1500 nm) have a high penetration power into the human body without causing damage, ${ }^{(14-16)}$ and scintillators emitting NIR photons are promising for use in radiation-based bioimaging applications. ${ }^{(17)}$ Moreover, scintillators emitting NIR photons are considered to work effectively in a high-dose environment. In general, the combination of a scintillator and optical fiber has been used for monitoring high radiation doses. However, optical fibers are damaged by high radiation doses, resulting in strong absorption of UV and visible photons. ${ }^{(17)}$ Moreover, Cherenkov radiation appears in the UV-blue range in a high-dose environment such as a nuclear

*Corresponding author: e-mail: akatsuka.masaki.ad5@ms.naist.jp https://doi.org/10.18494/SAM.2021.3319 
reactor. ${ }^{(18,19)}$ If scintillator materials emitting UV-blue light are used in the above situation, the Cherenkov radiation will overlap with scintillation signals, leading to incorrect radiation measurements. However, scintillators emitting NIR photons would be advantageous in such a measurement configuration because the NIR scintillation signal can be easily separated from the noise generated by Cherenkov radiation and less radiation damage will occur in the NIR range. Motivated by the above ideas, we have studied scintillating materials emitting NIR photons (700-1650 nm) including fluorides, sesquioxides, oxide garnets, and oxide perovskites. ${ }^{(20-23)}$

To expand the investigation on NIR-emitting scintillators, we synthesized Nd-doped $\mathrm{GdVO}_{4}$ single crystals by the floating zone (FZ) method and evaluated their photoluminescence (PL) and scintillation properties in this study. Nd-doped $\mathrm{GdVO}_{4}$ single crystals were studied because the vanadates are well-known phosphor and laser materials, ${ }^{(24-27)}$ and the scintillation properties of Nd-doped $\mathrm{YVO}_{4}$ have already been studied. ${ }^{(28)}$ However, to our knowledge, there have been no studies on the NIR scintillation properties of Nd-doped $\mathrm{GdVO}_{4}$.

\section{Materials and Methods}

$\mathrm{Gd}_{2} \mathrm{O}_{3}(4 \mathrm{~N}), \mathrm{V}_{2} \mathrm{O}_{5}(4 \mathrm{~N})$, and $\mathrm{Nd}_{2} \mathrm{O}_{3}(4 \mathrm{~N})$ were prepared as raw material powders to synthesize a series of Nd-doped $\mathrm{GdVO}_{4}$ samples. The nominal concentrations of $\mathrm{Nd}$ were 0.1, 0.3, 1.0, 3.0, and $10 \mathrm{~mol} \%$, and the $\mathrm{Gd}^{3+}$ site was substituted with $\mathrm{Nd}^{3+}$. A non-doped sample was also prepared for comparison. After mixing the powders, a cylindrical rubber balloon was filled with the powder mixture, and then the balloon was formed into a rod shape by hydrostatic pressure. To obtain a ceramic rod, the shaped rod was sintered at $1100{ }^{\circ} \mathrm{C}$ for $8 \mathrm{~h}$ in air. The obtained ceramic rod was grown into a single crystal in an FZ furnace (Canon Machinery FZD0192). During the growth, the rotation rate was $20 \mathrm{rpm}$ and the pull-down rate was $2.5-5.0 \mathrm{~mm} / \mathrm{h}$.

To obtain the crystal structure, powder X-ray crystallography was performed using an X-ray diffractometer (Miniflex 600, Rigaku). A microfocus X-ray tube was used, where the target material was $\mathrm{Cu}$. During the measurement, the X-ray tube voltage was set to $40 \mathrm{kV}$ and the current was $15 \mathrm{~mA}$.

A PL excitation and emission contour map (PL map) was obtained and the PL quantum yield $(Q Y)$ was measured using a Quantaurus-QY device (C11347, Hamamatsu). The contour map ranges for the PL excitation and emission were 250-800 and 300-950 nm, respectively, and the measurement interval for the excitation wavelength was $10 \mathrm{~nm}$. The PL decay time profile was obtained using a Quantaurus- $\tau$ device (C11367, Hamamatsu). Here, we selected 575-625 and 900 $\mathrm{nm}$ as the excitation and monitoring wavelengths, respectively.

The scintillation spectra of the single-crystal samples were obtained using an X-ray generator (XRB80N100/CB, Spellman) and a spectrometer (Andor DU492A, which covered the range of 650-1650 nm). The setup of this measurement system was reported previously. ${ }^{(29)}$ The scintillation decay time profile was obtained using an afterglow characterization system. ${ }^{\left({ }^{(0)}\right)}$ The PMT used in this system covered the spectral range from 400 to $900 \mathrm{~nm}$. The voltage applied to the pulse X-ray source was $40 \mathrm{kV}$. The obtained decay time curves of both the PL and scintillation were approximated by least-squares fitting with an exponential decay function. 
To evaluate the suitability of the samples for use as detectors, the relationship between the scintillation signal intensity and the X-ray exposure dose rate from 0.006 to $60 \mathrm{~Gy} / \mathrm{h}$ in the NIR range was determined using the measurement system shown in Fig. 1. The X-ray tube was supplied with a $40 \mathrm{kV}$ bias voltage and several different tube currents $(5.2,0.52$, and $0.052 \mathrm{~mA}$ ) to change the X-ray exposure dose rate. The InGaAs PIN photodiode (Hamamatsu Photonics, G12180-250A) used in this system covered the spectral range from 950 to $1700 \mathrm{~nm}$. The NIR photons emitted from the sample were guided to the InGaAs PIN photodiode through an optical fiber (Thorlabs, FP600ERT) with $5 \mathrm{~m}$ length and $600 \mu \mathrm{m}$ core diameter. The PIN photodiode was mounted on a heat dissipator (Hamamatsu Photonics, A3179) and was cooled to $253 \mathrm{~K}$ using a temperature controller (Hamamatsu Photonics, C1103) to reduce thermal noise. The electric signals from the PIN photodiode were measured using an ammeter (Keysight, B2985A).

\section{Results and Discussion}

After the crystal growth, crystal rods of typically $4 \mathrm{~mm}$ diameter and $20 \mathrm{~mm}$ length were obtained, which were broken into pieces for characterization. Figure 2 shows all the samples used in this study (from left to right, non-doped, 0.1, 0.3, 1.0, 3.0, and $10 \mathrm{~mol} \%$ ). The samples changed from colorless to purple with increasing $\mathrm{Nd}$ concentration.

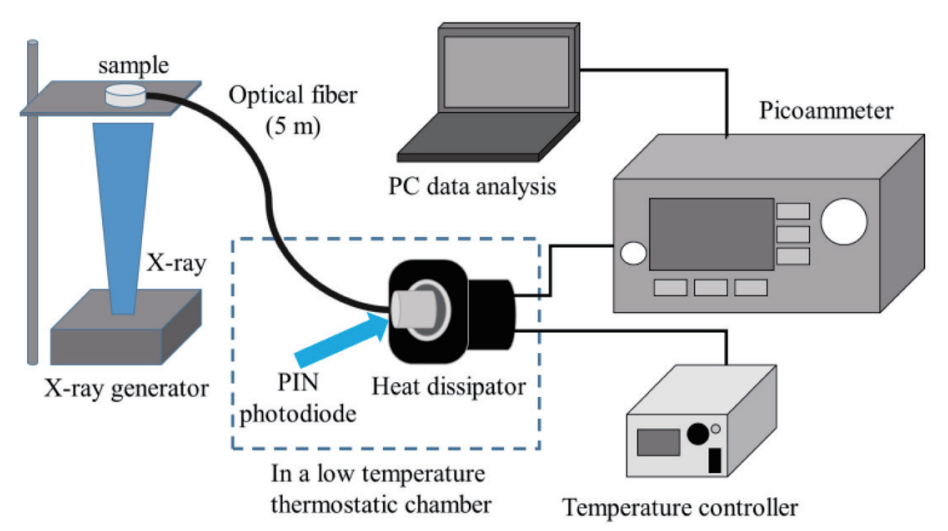

Fig. 1. (Color online) Schematic drawing of scintillation signal measurement system.

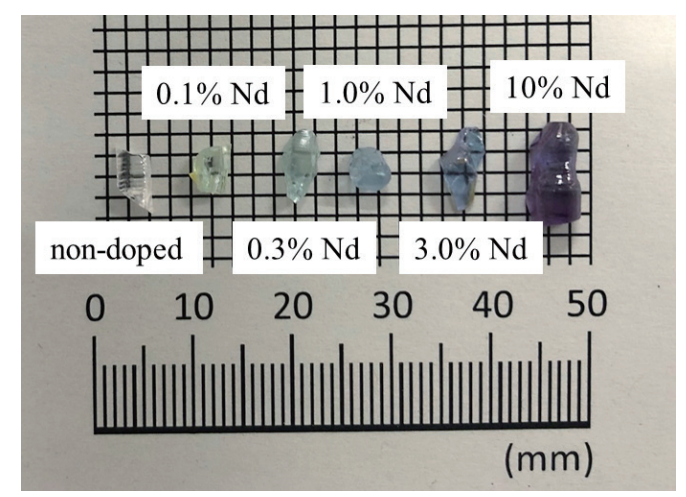

Fig. 2. (Color online) Photograph of $\mathrm{GdVO}_{4}$ samples doped with different concentrations of Nd. 
Figure 3 shows diffuse transmittance spectra of $\mathrm{GdVO}_{4}$ doped with different concentrations of $\mathrm{Nd}$. The diffuse transmittance of all the samples was $30-60 \%$ for wavelengths longer than $350 \mathrm{~nm}$. and the Nd-doped samples showed some absorption lines due to $4 \mathrm{f}-4 \mathrm{f}$ transitions of $\mathrm{Nd}^{3+} \cdot(31)$

Figure 4(a) shows powder X-ray diffraction (XRD) patterns of all the samples, with the diffraction pattern of $\mathrm{GdVO}_{4}$ (Inorganic Crystal Structure Database \#015607) also shown for comparison. Since the diffraction patterns of all the samples were in good agreement with the reference pattern, we confirmed that all the samples had the zircon-type structure, which belongs to the $\mathrm{I}_{1}$ /amd space group of a tetragonal crystal system. The enlarged diffraction patterns are shown in Fig. 4(b). The diffraction peaks around $25^{\circ}$ shift to smaller angles as the $\mathrm{Nd} / \mathrm{Gd}$ ratio increases, which is due to the increase in the lattice constant.

Figure 5 shows PL maps of the (a) non-doped sample and (b) $0.1 \mathrm{~mol} \% \mathrm{Nd}$-doped sample. The other Nd-doped samples also exhibited the same emission features. The PL map of the non-

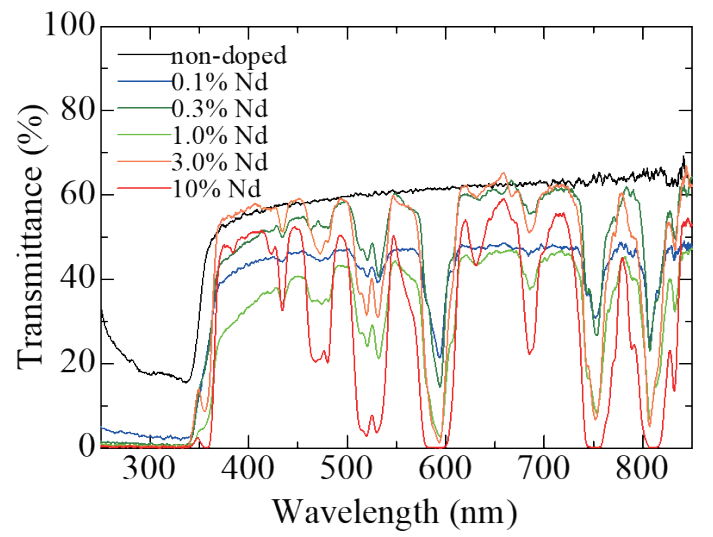

Fig. 3. (Color online) Diffuse transmittance spectra of $\mathrm{GdVO}_{4}$ doped with different concentrations of Nd.
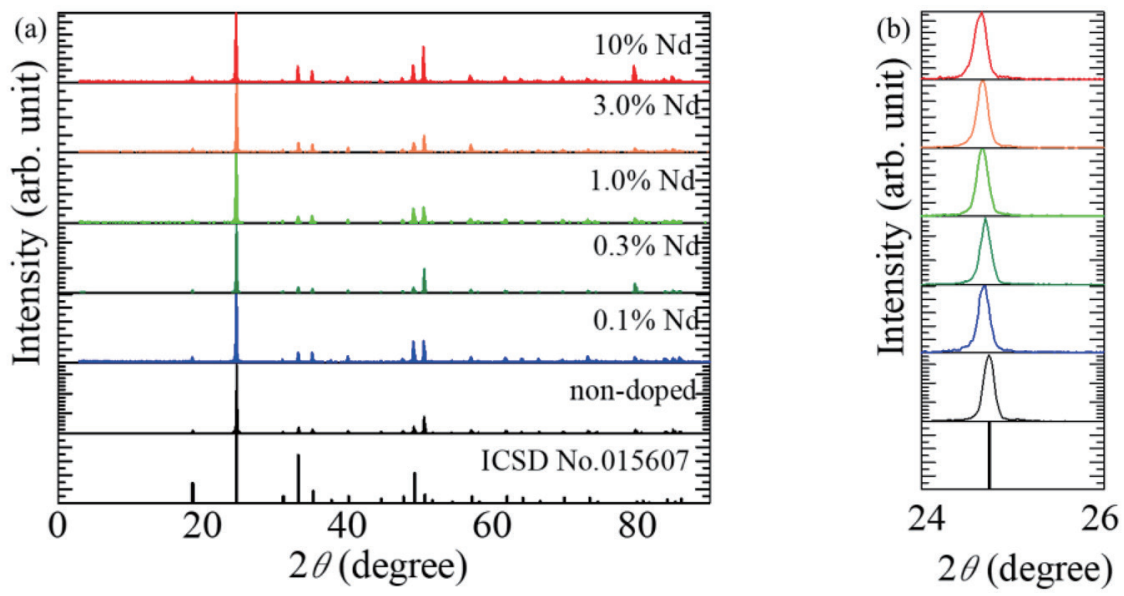

Fig. 4. (Color online) (a) Powder XRD patterns of $\mathrm{GdVO}_{4}$ crystals doped with different concentrations of $\mathrm{Nd}$ and (b) enlarged view of patterns in the range from 24 to $26^{\circ}$. 

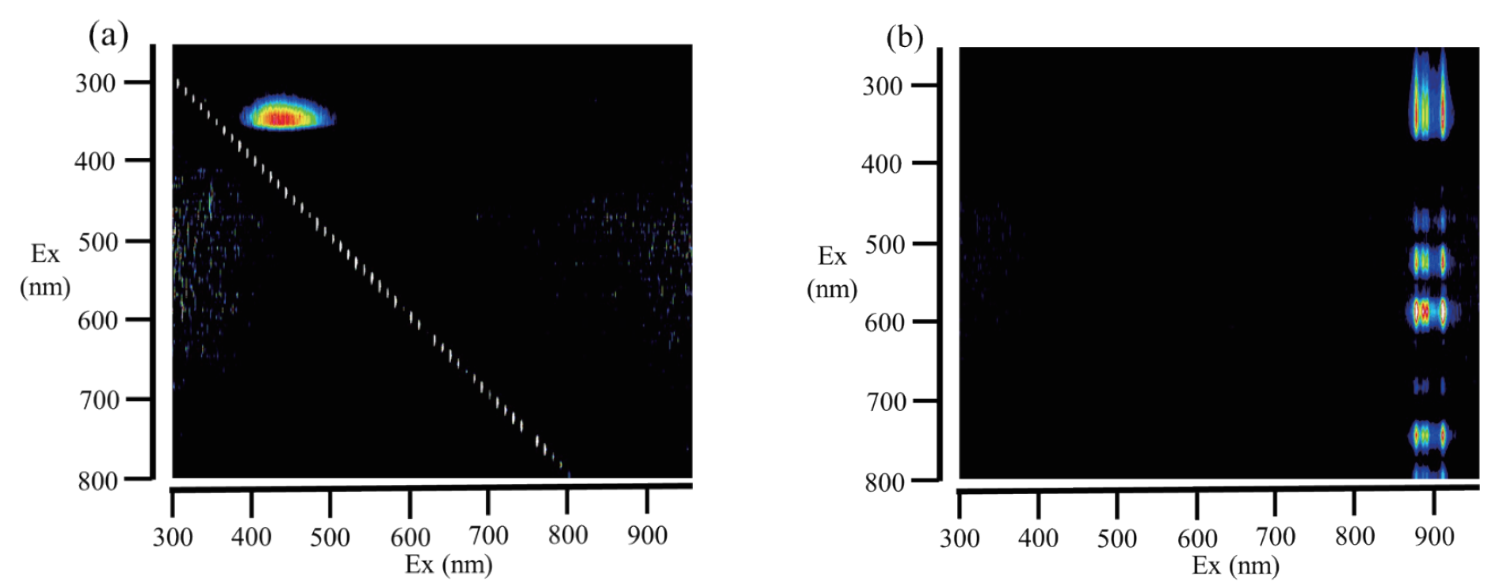

Fig. 5. (Color online) PL maps of (a) non-doped and (b) $0.1 \mathrm{~mol} \%$ Nd-doped samples. The horizontal and vertical axes indicate the emission and excitation wavelengths, respectively.

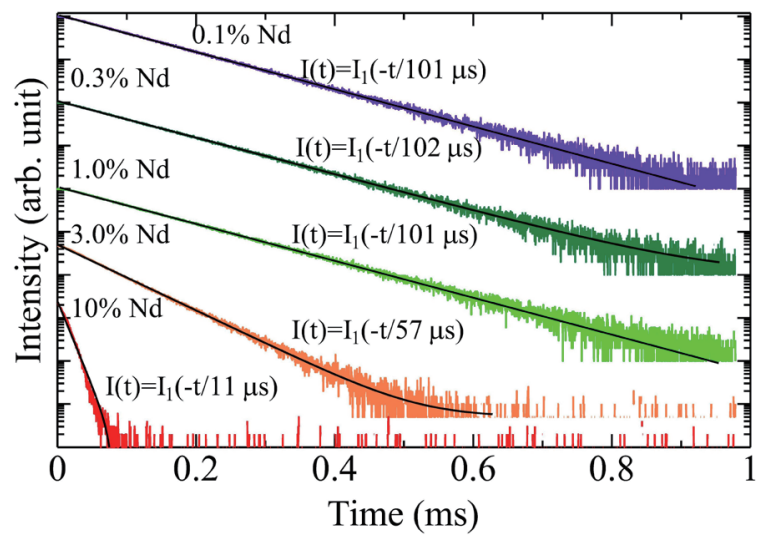

Fig. 6. (Color online) PL decay curves of Nd-doped samples. The excitation and monitoring wavelengths were $575-625$ and $810 \mathrm{~nm}$, respectively.

doped sample showed emission around 400-500 $\mathrm{nm}$ due to the transition from triplet states of $\mathrm{VO}_{4}{ }^{3-} \cdot(32,33)$ As shown in Fig. 4(b), strong emission was confirmed around $900 \mathrm{~nm}$ owing to the $4 \mathrm{f}-4 \mathrm{f}$ transitions of $\mathrm{Nd}^{3+} \cdot{ }^{34,35)}$ The $Q Y$ values of the Nd-doped samples were calculated by integrating the signal intensity from 800 to $950 \mathrm{~nm}$ in order to focus on the NIR emission. The $Q Y$ values of the $0.1,0.31 .0,3.0$, and $10 \mathrm{~mol} \% \mathrm{Nd}$-doped samples were found to be 27.2, 20.9, $23.3,3.3$, and $0.7 \%$ with typical errors of $\pm 2 \%$, respectively. The $Q Y$ value was much lower for the $3.0 \mathrm{~mol} \% \mathrm{Nd}$-doped sample than for the $1.0 \mathrm{~mol} \% \mathrm{Nd}$-doped sample. Thus, concentration quenching may have arisen at a $\mathrm{Nd}$ concentration of around $3.0 \mathrm{~mol} \%$.

PL decay curves of the Nd-doped samples are shown in Fig. 6. Here, the excitation and monitoring wavelengths were 575-625 and $810 \mathrm{~nm}$, respectively. All the decay curves followed single-component simple exponential decay. For the $0.1-1.0 \mathrm{~mol} \% \mathrm{Nd}$-doped samples, the PL decay times were around $100 \mu \mathrm{s}$. These decay times are typical for the $4 \mathrm{f}-4 \mathrm{f}$ transitions of $\mathrm{Nd}^{3+}$ 
and agree with previously reported values. ${ }^{(36)}$ In contrast, the decay times of the 3.0 and 10 mol\% Nd-doped samples were lower. This is considered to be due to concentration quenching of samples, and this tendency was consistent with the $Q Y$ values.

Figure 7 shows the X-ray-induced scintillation spectra of all the samples in the NIR range with the intensity normalized. All the Nd-doped samples exhibited three emission peaks at around 900, 1060, and $1320 \mathrm{~nm}$. The intensity of the emission peak around $1060 \mathrm{~nm}$ was the highest, and this emission is commonly used in laser applications. ${ }^{(37)}$ These emissions were due to the electronic transitions of $\mathrm{Nd}^{3+}{ }^{4} \mathrm{~F}_{3 / 2} \rightarrow{ }^{4} \mathrm{I}_{9 / 2}(900 \mathrm{~nm}),{ }^{4} \mathrm{~F}_{3 / 2} \rightarrow{ }^{4} \mathrm{I}_{11 / 2}(1060 \mathrm{~nm})$, and ${ }^{4} \mathrm{~F}_{3 / 2} \rightarrow{ }^{4} \mathrm{I}_{13 / 2}(1320 \mathrm{~nm}){ }^{(32,38,39)}$

The X-ray-induced scintillation decay time profiles of the Nd-doped samples are illustrated in Fig. 8. To determine the decay time constants, the decay curves of all the Nd-doped samples were approximated by the sum of two exponential decay functions. The first decay component was regarded to be a tail due to the instrumental response $(\sim 1.8 \mu \mathrm{s})$, and the second decay component was considered to be the signal from the sample. The decay times of the $0.1-1.0$ mol\% Nd-doped samples were typical values for the $4 \mathrm{f}-4 \mathrm{f}$ transitions of $\mathrm{Nd}^{3+},(40,41)$ and the 3.0 and $10 \mathrm{~mol} \% \mathrm{Nd}$-doped samples had very short decay times. These tendencies were similar to those of the PL. For these reasons, the origin of the emission of scintillation was ascribed to the 4f-4f transitions of $\mathrm{Nd}^{3+}$, and it was concluded that the 3.0 and $10 \mathrm{~mol} \% \mathrm{Nd}-$ doped samples suffered from concentration quenching.

To evaluate the suitability of the samples for use as detectors, the relationship between the average scintillation signal intensity from 960 to $1700 \mathrm{~nm}$ and the X-ray exposure dose rate was evaluated in the dose rate range from 0.006 to $60 \mathrm{~Gy} / \mathrm{h}$. The obtained results are shown in Fig. 9. All the Nd-doped samples exhibited high sensitivity in the dynamic range from 0.006 to 60 Gy/h.

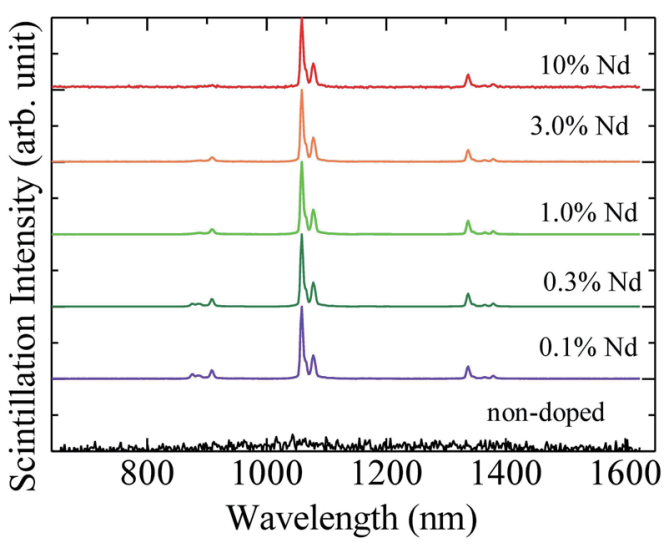

Fig. 7. (Color online) X-ray induced scintillation properties of all samples in the NIR range. The intensities are normalized.

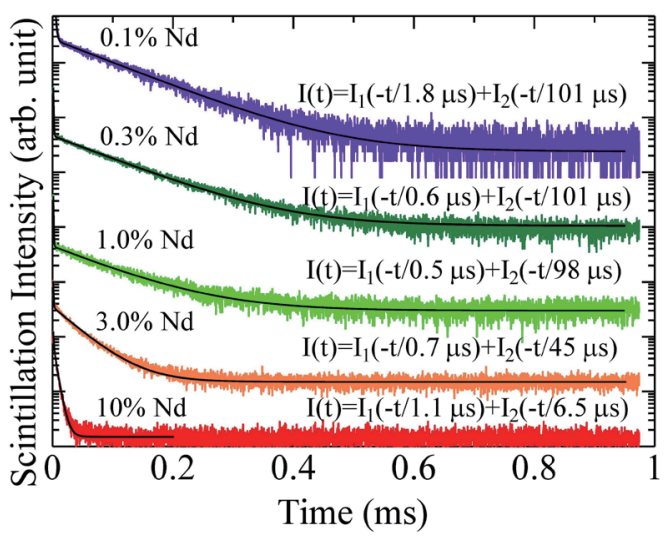

Fig. 8. (Color online) X-ray-induced scintillation decay curves of Nd-doped samples. 


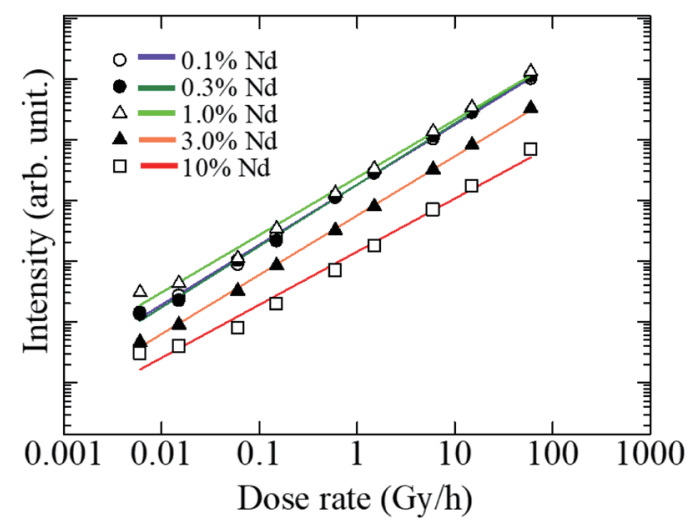

Fig. 9. (Color online) Relationship between scintillation signal intensity and X-ray exposure dose rate of Nddoped $\mathrm{GdVO}_{4}$ shown on log-log scale. The scintillation signal range is from 950 to $1700 \mathrm{~nm}$ and the exposure range is from 0.006 to $60 \mathrm{~Gy} / \mathrm{h}$.

\section{Conclusions}

A series of $\mathrm{GdVO}_{4}$ single crystals doped with different concentrations of $\mathrm{Nd}$ were synthesized by the FZ method, and their PL and scintillation properties were evaluated. According to its PL map, the non-doped sample exhibited emission around 400-500 $\mathrm{nm}$ due to the transition from the triplet state of $\mathrm{VO}_{4}{ }^{3-}$, and all $\mathrm{Nd}$-doped samples showed emissions around $900 \mathrm{~nm}$ due to the $4 \mathrm{f}-4 \mathrm{f}$ transitions of $\mathrm{Nd}^{3+}$. The PL decay curves of the Nd-doped samples could be approximated by a single exponential decay function. The X-ray-induced scintillation spectra of all the Nddoped samples exhibited emission peaks at around 900, 1060, and $1320 \mathrm{~nm}$. To evaluate the potential of the doped single crystals for dosimetric applications, the relationship between the scintillation signal intensity and the X-ray exposure dose rate was evaluated from 0.006 to 60 $\mathrm{Gy} / \mathrm{h}$ in the NIR range, and the Nd-doped samples exhibited high sensitivity from 0.006 to 60 $\mathrm{Gy} / \mathrm{h}$.

\section{Acknowledgments}

This work was supported by Grants-in-Aid for Scientific Research (A) (17H01375), (B) (18H03468 and 19H03533), and Japan Society for the Promotion of Science (JSPS) Research Fellowship (19J22402) from the Ministry of Education, Culture, Sports, Science and Technology of the Japanese government (MEXT). The Cooperative Research Project of the Research Center for Biomedical Engineering, Murata Foundation, Nippon Sheet Glass Foundation, Iketani Foundation, and NAIST Foundation are also acknowledged.

\section{References}

1 T. Yanagida: Proc. Japan Acad. Ser. B. 94 (2018) 75.

2 J. Glodo, Y. Wang, R. Shawgo, C. Brecher, R. H. Hawrami, J. Tower, and K. S. Shah: Phys. Procedia 90 (2017) 285. 
3 S. Yamamoto, K. Kuroda, and M. Senda: IEEE Nucl. Sci. Symp. Conf. Rec. (IEEE, 2002) 1632.

4 M. Kokubun, K. Abe, Y. Ezoe, Y. Fukazawa, S. Hong, H. Inoue, T. Itoh, T. Kamae, D. Kasama, M. Kawaharada, N. Kawano, K. Kawashima, S. Kawasoe, Y. Kobayashi, J. Kotoku, M. Kouda, A. Kubota, G. M. Madejski, K. Makishima, T. Mitani, H. Miyasaka, R. Miyawaki, K. Mori, M. Mori, T. Murakami, M. M. Murashima, K. Nakazawa, H. Niko, M. Nomachi, M. Ohno, Y. Okada, K. Oonuki, G. Sato, M. Suzuki, H. Takahashi, I. Takahashi, T. Takahashi, K. Tamura, T. Tanaka, M. Tashiro, Y. Terada, S. Tominaga, S. Watanabe, K. Yamaoka, T. Yanagida, and D. Yonetoku: IEEE Trans. Nucl. Sci. 51 (2004) 1991.

5 S. Moriuchi, M. Tsutsumi, and K. Saito: Jpn. J. Heal. Phys. 44 (2009) 122.

6 T. Yanagida, Y. Fujimoto, M. Arai, M. Koshimizu, T. Kato, D. Nakauchi, and N. Kawaguchi: Sens. Mater. 32 (2020) 1351.

7 P. Kantuptim, M. Akatsuka, D. Nakauchi, T. Kato, N. Kawaguchi, and T. Yanagida: Sens. Mater. 169 (2019) 106401.

8 H. Kimura, T. Kato, D. Nakauchi, N. Kawaguchi, and T. Yanagida: Sens. Mater. 32 (2020) 1381.

9 D. Shiratori, D. Nakauchi, T. Kato, N. Kawaguchi, and T. Yanagida: Sens. Mater. 32 (2020) 1365.

10 N. Kawaguchi and T. Yanagida: Sens. Mater. 31 (2019) 1257.

11 M. Koshimizu, T. Yanagida, R. Kamishima, Y. Fujimoto, and K. Asai: Sens. Mater. 31 (2019) 1233.

12 Y. Araya, M. Koshimizu, R. Haruki, F. Nishikido, S. Kishimoto, and K. Asai: Sens. Mater. 27 (2015) 255.

13 A. Horimoto, N. Kawano, D. Nakauchi, H. Kimura, M. Akatsuka, and T. Yanagida: Sens. Mater. 32 (2020) 1395.

14 J. L. Boulnois: Lasers Med. Sci. 1 (1986) 47.

15 L. Sudheendra, G. K. Das, C. Li, D. Stark, J. Cena, S. Cherry, and I. M. Kennedy: Chem. Mater. 26 (2014) 1881.

16 K. Soga, T. Tsuji, F. Tashiro, J. Chiba, M. Oishi, K. Yoshimoto, Y. Nagasaki, K. Kitano, and S. Hamaguchi: J. Phys. Conf. Ser. 106 (2008) 012023.

17 K. Toh, T. Nakamura, H. Yamagishi, K. Sakasai, K. Soyama, and T. Shikama: Nucl. Instrum. Methods Phys. Res., Sect. A 700 (2013) 130.

18 J. Archer, L. Madden, E. Li, M. Carolan, and A. Rosenfeld: Biomed. Phys. Eng. Express 4 (2018) 044003.

19 A. S. Beddar, T. R. Mackie, and F. H. Attix: Phys. Med. Biol. 37 (1992) 1901.

20 T. Yanagida, Y. Fujimoto, H. Yagi, and T. Yanagitani: Opt. Mater. 36 (2014) 1044.

21 M. Akatsuka, D. Nakauchi, T. Kato, N. Kawaguchi, and T. Yanagida: Sens. Mater. 32 (2020) 1373.

22 T. Yanagida, Y. Fujimoto, S. Ishizu, and K. Fukuda: Opt. Mater. 41 (2015) 36.

23 G. Okada, N. Kawaguchi, and T. Yanagida: Sens. Mater. 29 (2017) 1407.

24 B. Yan and X. Q. Su: Opt. Mater. 29 (2007) 547.

25 J. H. Kang, W. Bin Im, D. C. Lee, J. Y. Kim, D. Y. Jeon, Y. C. Kang, and K. Y. Jung: Solid State Commun. 133 (2005) 651.

26 M. Higuchi, H. Sagae, K. Kodaira, T. Ogawa, S. Wada, and H. Machida: J. Cryst. Growth 264 (2004) 284.

27 M. Soharab, I. Bhaumik, R. Bhatt, A. Saxena, S. Khan, and A. K. Karnal: Opt. Mater. 92 (2019) 379.

28 Y. Fujimoto, T. Yanagida, T. Kojima, M. Koshimizu, H. Tanaka, and K. Asai: Sens. Mater. 28 (2016) 857.

29 T. Yanagida, K. Kamada, Y. Fujimoto, H. Yagi, and T. Yanagitani: Opt. Mater. 7 (2013) 2480.

30 T. Yanagida, Y. Fujimoto, T. Ito, K. Uchiyama, and K. Mori: Appl. Phys. Express 7 (2014) 062401.

31 S. Möller, A. Hoffmann, D. Knaut, J. Flottmann, and T. Jüstel: J. Lumin. 158 (2015) 365.

32 L. Yang, C. Wang, Y. Dong, N. Da, X. Hu, D. Chen, and J. Qiu: Opt. Express 13 (2005) 10157.

33 K. Oka, H. Unoki, H. Shibata, and H. Eisaki: J. Cryst. Growth 286 (2006) 288.

34 J. Lu, J. Lu, T. Murai, K. Takaichi, T. Uematsu, K. I. Ueda, H. Yagi, T. Yanagitani, and A. A. Kaminskii: Jpn. J. Appl. Phys. 40 (2001) L1277.

35 M. A. Hernández-Rodríguez, A. D. Lozano-Gorrín, I. R. Martín, U. R. Rodríguez-Mendoza, and V. Lavín: Sens. Actuators, B 255 (2018) 970.

36 H. Yagi, T. Yanagitani, K. Takaichi, K. Ueda, and A. A. Kaminskii: Opt. Mater. 29 (2007) 1258.

37 J. Wang, Y. Zhang, H. Xia, and J. Sheng: Bull. Mater. Sci. 32 (2009) 183.

38 T. Oya, G. Okada, and T. Yanagida: J. Ceram. Soc. Jpn. 124 (2016) 536.

39 M. Akatsuka, Y. Usui, D. Nakauchi, G. Okada, N. Kawaguchi, and T. Yanagida: Sens. Mater. 30 (2018) 1525.

40 M. Akatsuka, N. Kawaguchi, and T. Yanagida: J. Ceram. Process. Res. 20 (2019) 280.

41 T. Yanagida, M. Akatsuka, G. Okada, and N. Kawaguchi: Opt. Mater. 90 (2019) 14. 